Canadian University Music Review

Revue de musique des universités canadiennes

\title{
L'Action dans le récitatif pathétique de Lully : la déclamation
}

\section{Jean-Pierre Pinson}

Numéro 5, 1984

URI : https://id.erudit.org/iderudit/1013938ar

DOI : https://doi.org/10.7202/1013938ar

Aller au sommaire du numéro

Éditeur(s)

Canadian University Music Society / Société de musique des universités

canadiennes

ISSN

0710-0353 (imprimé)

2291-2436 (numérique)

Découvrir la revue

Citer cet article

Pinson, J.-P. (1984). L’Action dans le récitatif pathétique de Lully : la déclamation. Canadian University Music Review / Revue de musique des universités canadiennes, (5), 152-178. https://doi.org/10.7202/1013938ar

All Rights Reserved (C Canadian University Music Society / Société de musique des universités canadiennes, 1984
Ce document est protégé par la loi sur le droit d'auteur. L'utilisation des services d'Érudit (y compris la reproduction) est assujettie à sa politique d'utilisation que vous pouvez consulter en ligne.

https://apropos.erudit.org/fr/usagers/politique-dutilisation/ 


\title{
L'ACTION DANS LE RÉCITATIF PATHÉTIQUE DE LULLY : LA DÉCLAMATION
}

\author{
Jean-Pierre Pinson
}

\begin{abstract}
"À l'égard de l'Action, on peut en donner les règles, et instruire ceux qui l'apprennent, en leur donnant des avis, ou en déclamant devant eux; mais il n'est pas possible de leur rien apprendre comme il faut sur le papier. " (Gibert, La rhétorique ou les règles de l'éloquence, 1730).
\end{abstract}

L'opéra de Lully et en particulier son récitatif ne sont encore aujourd'hui pour ainsi dire jamais joués. Comme le disaient déjà les étrangers de passage à l'Académie de Musique, cela tient sans doute à ce qu'on n'y chante pas assez. Remond de Saint-Mard résumait ainsi la question :

L'Opéra est presqu'une Tragédie dans les formes, une Tragédie mise en Musique, en conséquence de quoi il faut qu'il soit joué, chanté et déclamé. Or de ces trois choses là, il me semble qu'on n'y en fait qu'une : on y chante, et encore y chante-t-on assez mal; pour le reste il en est très-peu question, et voilà pourquoi tel Opéra, qui pourroit être charmant, réussit quelquefois à nous ennuyer (1741:70).

Le but de cet article est de résumer, à l'usage de nos chanteurs qui chantent trop, les règles de l'action, c'est-à-dire de la déclamation, telles qu'on les trouve dans les principaux traités. J'utiliserai comme exemple le grand récitatif et air d'Armide, placé à la fin du deuxième acte de l'opéra du même nom (1686) (voir en annexe). Il ne sera pas question de considérations historiques (l'évolution du récitatif), des querelles, de la manière italienne, du bien-fondé esthétique, des défauts des acteurs. Je propose plutôt une perspective pratique : relever un vocabulaire. Ce qui suit n'a rien d'original, sinon que je classe les moyens selon une démarche concrète : construire un personnage et le jouer avec cohérence. 


\section{Le discours pathétique de l'acteur-chanteur}

Le monde classique français est le monde de la parole, où se mêlent les arts de la conversation (qui a ses manuels), de la lecture, de l'éloquence, du théâtre, du récitatif et du chant. Grimarest, dans son Traité du récitatif (1707-1760), décrit ainsi tour à tour la lecture, "l'action publique " (l'éloquence), la déclamation théâtrale et le chant. Le jeu des citations prouve combien les français sont autant sensibles à la musique qu'au pouvoir qu'elle a d'« outrer la voix », de déclamer :

"Le Chant n'est qu'un parler agréable, inventé pour ajouter de la force aux paroles" (Michel de Pure 1668).

«Il faut chanter comme on parle " (Bénigne de Bacilly 1679).

"Faire de la musique c'est faire parler en chant un homme " (Le Cerf de la Viéville 1705-1706).

"Le récitatif doit se rapprocher le plus qu'il est possible de la déclamation" (Pierre Estève 1753).

C'est aussi pourquoi les auteurs qui nous décrivent la tradition de la déclamation théâtrale soulignent le mouvement inverse de la parole vers la musique. Ainsi Estève rapporte que "pendant longtemps il y a eu sur le théâtre français une déclamation chantante " (1753: 134). D’après Claude-Joseph Dorat, " $M^{\text {lle }}$ Duclos (...) introduisait dans la déclamation une espèce de Musique et de chant (...) Elle déclamait par octave, et l'on aurait pu noter ses inflexions » (1766:16). L'exemple de Lully allant écouter la Champmeslé, interprète et élève de Racine, celui de Voltaire tirant des larmes à ses auditeurs (dit-il) à la seule lecture de Quinault, et selon les inflexions du récitatif de Lully $^{1}$, tout cela est bien connu.

À l'opéra, le bon chanteur est aussi un bon comédien, c'est-à-dire un bon déclamateur, qui s'inspire de la déclamation théâtrale et rhétorique : Grimarest est catégorique : " lorsqu'un bon comédien a la voix favorable pour le chant, il l'exécute à la satisfaction de ceux qui l'écoutent " (1760:38). À cet acteurchanteur (Jean-Jacques Nattiez [1983] propose l'expression "chantacteur") revient le rôle de déclamer ce que ces temps littéraires appelèrent tour à tour "tragédie en musique », puis "tragédie », puis "tragédie lyrique », et à nouveau "tragédie en musique ". Dans cet univers baigné de rhétorique, on apprend, dans les classes terminales des collèges, à déclamer, donc à suivre les règles de l'action, dernière des quatre parties de la 
rhétorique. Comment cette rhétorique n'aurait-elle pas influencé aussi le jeu théâtral ?

Bien sûr, les auteurs sont conscients des spécificités de chacun des arts de la parole. Mais il reste cette obsession du discours. L'abbé d'Aubignac, dans sa Pratique du théâtre, renvoie son lecteur aux nombreux ouvrages de rhétorique parus depuis Aristote, et se réserve le discours théâtral :

Je considère au Théâtre quatre sortes de Discours. Les Narrations, les Délibérations, les Discours Didactiques ou Instructions, et les Discours Pathétiques ou mouvements et Passions (1715: 264).

On reconnaîtra ici les dominantes du discours oratoire, tel que défini par Quintilien et Cicéron, et repris par l'humanisme des $\mathrm{XVI}^{\mathrm{e}}$ et XVII siècles, et le théâtre de Corneille (cf. en particulier Lazare [1980] et Scherer [1950]). Comme le rôle de l'opéra est d'émouvoir et d'étonner, il donnera sa préférence au discours pathétique. C'est pourquoi j'analyserai ici non pas le récitatif "simple", "ordinaire", destiné à faire avancer l'action, réservé aux "moments dénués d'intérêt " (Lacépède 1785 : II, 48), donc à la simple information. Je considérerai plutôt le " récitatif psychologique », dont Lully donne les premiers exemples², et que ses successeurs, jusqu'à Gluck, vont chercher à enrichir.

Ce récitatif apparaît le plus souvent dans les monologues, qui sont au coeur de l'action du théâtre classique. Comme au théâtre, ce récitatif relève de cette "extension de la forme linguistique d'exclamation; c'est la parole expressive par excellence qui ne s'adresse à aucun interlocuteur", disait Tzvetan Todorov (rapporté par Larthomas 1980 : 374). C'est ce que Joyce Newman décrit comme le "recitative-monologue ", par opposition au "recitative-dialogue » et au " scene-complex ", qui met en scène plusieurs personnages, et souvent les danseurs et le chour (Newman 1979).

Ce monologue est la manifestation parfaite de ce que Jean Duvignaud (1970) décrit comme le discours d'un personnage délibérant sur son destin, à l'intérieur d'un espace clos, celui de la scène à l'italienne. Ce discours est intense, donc plutôt rare dans l'ouvrage. Sa place est soigneusement choisie dans l'action, puisqu'il décide d'un destin, ou y contribue. Jean-Louis Barrault souligne bien ce moment exceptionnel :

Loin maintenant de la réalité, nous entrons dans le monde spécifiquement poétique, dans le monde du rêve-éveillé. 
Le moment où l'homme se met à "penser à haute voix "; le moment où l'exaltation est si tendue que, poussée jusqu'au cas limite, elle pourrait s'exprimer par le chant (1946:54).

$\mathrm{Au}$ théâtre, comme dans l'opéra de Lully, ce discours pathétique est une pose essentielle, le point focal. Il est théâtre dans le théâtre, et rassemble les règles de la dramaturgie classique, comme celles de la stratégie rhétorique. Il est le lieu de ce que Larthomas appelle "la concentration des effets " et qui convient bien à ce « dialogue solitaire » du monologue-récitatif :

Nous entendons par là tout élément qui, dans un dialogue (là encore au sens le plus large du mot) se révèle utile, d'une manière ou d'une autre, parce que susceptible d'amener une réaction, si faible soit-elle, du spectateur (1980:281).

Armide, au deuxième acte de l'opéra, contemple son ennemi Renaud endormi, va pour le tuer, et en tombe amoureuse. Dix vers seulement suffisent. Il y a bien là une concentration des effets, c'est-à-dire des moyens dont disposaient les auteurs et l'interprète. Il s'agit maintenant de décrire et de classer ces moyens.

\section{L'invention}

L'invention, première partie de la rhétorique, est l'art de "trouver ce dont on va parler ". C'est la quête des preuves du discours judiciaire, du sujet d'un poème. C'est la recherche des lieux communs, rassemblés dans le réservoir de la Topique. À cette époque qui fonde son esthétique sur l'imitation de la belle nature, les peintres, les poètes et les musiciens représentent une "nature idéale », dans des "tableaux », des "portraits " idéaux. Sous le particulier, on cherche le général, le type. Et le catalogue des types est restreint, plus encore à l'opéra qu'au théâtre. Le type d'Armide est celui de ces jeunes héros et héroïnes guerrières (Tancrède, Clorinde, Roland de Quinault et Lully). Ces personnages sont beaux, fougueux, impétueux, prompts à la colère et à l'amour. Comme le spectacle de l'opéra leur interdit une psychologie complexe, racinienne, ils tiennent beaucoup de l'allégorie (cf. McGowan 1978). Une mise en scène et une gestique hiératique (cf. Barnett 1977/78), de lourds costumes en font des acteurs-statues dont l'interprétation ne pouvait être que hautement stylisée. Il leur faut donc un certain nombre d'attributs, et de passions nettement délimitées. Comme Médée - des mêmes Quinault et Lully - Armide éprouve la 
colère, la "rage ", la "furor " de ces héros emportés. Mais elle succombera à la pitié, l'amour et la tendresse. En dix vers, elle passe en revue les deux principales catégories de passions qu'Aristote rangeait soit du côté de la terreur soit du côté de la pitié, et que les théoriciens allemands de l'Affektenlehre, depuis la Renaissance, aimaient classer en "passions tranquilles " ou "bons sentiments " (" affectus boni ») et en "passions agitées», ou "mauvais sentiments": telles sont la piacevolezza et la vehemanza du Tasse que Monteverdi reprend dans ses Madrigali guerrieri et amorosi. Armide, créature de Quinault, auteur français classique et modéré, démontre le passage du pathos à l'ethos, domaine des sentiments tendres, dont l'air de cour avait gardé la tradition. Personnification de la colère, Armide, brusquement, change d'attributs : la redoutable magicienne orientale (allégorie de l'Asie ${ }^{3}$ ) se soumet à l'éthos de la civilisation européenne, représentée par Renaud, et la tendresse qu'elle éprouve pour lui.

\section{L'élocution}

Dans la première et seconde rhétorique - ou poétique l'élocution est l'autre nom de la stylistique. Car il faut mettre en forme cette peinture des passions dont la parole devra rendre compte. Le mot, avant la musique, est le moyen terme entre la chose imitée et celui qui la contemple. Snyders a bien décrit ce rôle intermédiaire du mot, placé à mi-distance " entre la musique et son modèle " démontrant que "la musique va devenir une peinture au second degré, imitant non pas directemet le réel, mais les mots dans lequel le réel se trouve déjà inclus " (1968:20). L'élocution aura pour rôle stylistique de veiller à l'adéquation entre la nature et les signes (gestuels, verbaux, musicaux). Tout en sachant bien qu'il ne peut y avoir une constante, rigoureuse et définitive correspondance, les auteurs les plus lucides se rendent cependant bien compte que cette esthétique entraîne une rigoureuse sélection du vocabulaire (littéraire et musical) : « (...) tel doit être le dessin des choses qu'il exprime l'idée même des choses " dit lui-aussi Nicolas Poussin. Il y a donc limitation des moyens, selon ce qu'on pourrait appeler la règle de la bienséance : " on pourrait dire de la poétique classique que c'est la poétique des bienséances" (Bray 1966 : 230).

La bienséance contraint le discours pathétique non seulement à respecter les règles de l'imitation (théorie du decorum, vraisemblance, poétique des styles et des genres, règles du goût), 
mais aussi, à l'autre bout de la chaîne, à prendre en considération la "théorie des mours ». Ainsi la morale, les "décences" contrôlaient la scène et le vocabulaire. "L'utilité morale " devait servir le «bien public ", la " tempérance " devait faire respecter la "civilité » d'un auditoire de qualité. Pas de mots "sales », d'actions "rebutantes".

De même, et par conséquent, l'orateur ou le comédien doivent, pour convaincre, faire preuve, dans leur attitude, de "probité », de "vertu », de "prudence », de "modestie " même (Lamy 1699 : 111). L'aspect physique et moral de l'acteur doit convenir au personnage. C'est pourquoi Titon du Tillet, décrivant Marie Rochois, créatrice du rôle d'Armide, s'émerveille du talent de cette actrice qui réussit à faire oublier son physique :

Quoiqu'elle fût d'une taille médiocre, fort brune, et d'une figure très commune hors du Théâtre (...) elle effaçoit toutes les plus belles actrices et les mieux faites quand elle étoit au Théâtre; elle avoit un air de Reine et de Divinité (1727 : 791).

C'est que nous sommes à une époque où les types d'acteurs doivent avoir des ressemblances avec les types de personnages qu'ils jouent : telle est la théorie des " emplois », pour lesquels il faut des dispositions.

Remond de Sainte-Albine écrit ainsi que "les personnes nées pour aimer, devroient avoir seules le privilège de jouer les rôles d'amants " (1749: 100). À l'opéra, la voix même entre dans cette esthétique de la bienséance : " chacun doit se servir de la voix que la nature lui a donnée, et ne jamais chercher à lui substituer un son qui n'est pas le sien propre », énonce François Riccoboni (1750:16).

À l'autre bout de la chaîne (la poïétique), la bienséance devient la convenance du vocabulaire. Quinault cherche les mots expressifs. Le monologue d'Armide est plein de " charme ", " percer ", "frappons ", " soupirs ", tous mots qui, par leur sens et leur prononciation, obéissent à la règle de l'imitation que Morellet (1759) a bien décrite. Suivent alors les autres règles de la vérité (de l'imitation), de la clarté, de la propriété (pas de confusion entre les termes), du mouvement même (à chaque " humeur » ou passion convient la juste expression dynamique). Voilà le dictionnaire des signes bien limité. Remond de SaintMard, étudiant les livrets des opéras de Lully, constate :

On y a [sic] environ que douze ou quinze cens mots, qu'il faut sans cesse tourner et retourner, et il n'y est guère possible d'en employer d'autres $(1741: 26)$. 
Le Cerf de la Viéville souligne bien les conséquences de cette rareté du vocabulaire sur le langage musical :

Quinault a donné cent fois à Lulli les mêmes sentimens et les mêmes termes à mettre en chant. Il n'est pas possible qu'il y ait cent manieres de les y mettre également bonnes (...) il a mieux aimé varier un peu moins ses tons que d'en employer de méchants (1705, troisième dialogue : 163).

Telle est donc cette esthétique de la densité, de l'économie : qui chercherait à retrancher du superflu au monologue d'Armide serait bien en peine. L'interprète n'en finira pas de repasser, de soupeser chacun des termes, avec sa densité sonore et son sens, comme avec la beauté du style et la suavité des mots : Remond de Saint-Mard, qu'on vient de citer, explique aussi la rareté des mots "par leur douceur et leur facilité ". Les règles du goût resserrent elles aussi les moyens. Lorsqu'Armide brandit son poignard, elle doit le faire avec une certaine grâce, de la simplicité, de la "finesse », de la " naïveté " même, dans le sens que Chiflet recommandait pour "refuir toute affection" du style (1659: 148). C'est, selon Formey «le langage de la franchise, de la liberté, de la simplicité [qui] comprend la chaleur, l'énergie, la vivacité " (1763:170-171). Telle est cette esthétique de la voluptas, pleine d'élégance jusque dans l'emportement, de politesse, jusque dans les gémissements. C'est ce que Le Cerf de la Viéville entend par " une musique naturelle, expressive, harmonieuse " (1705, quatrième dialogue : 301). C'est cette qualité du texte de Quinault que Marie Rochois devait faire sentir dans "toutes ses actions belles, justes et naturelles " (Titon du Tillet $1727: 791$ ).

Cependant, si Quinault et Lully s'en étaient tenus à ces règles contraignantes, nous n'aurions eu qu'une Armide académique. Le lecteur a remarqué le jeu des oppositions de termes ("vengeons-nous ... je soupire »). C'est que, pour donner vie à ce monologue pathétique, Quinault, au-delà du simple vocabulaire, joue avec la notion des styles et des genres.

Armide, belle magicienne, "sœur " de Circé, surprend Renaud sur les bords de l'Oronte, fleuve de Syrie : nous sommes en pleine tradition pastorale, italienne et française. Le thème des amours de Renaud va remplir l'imagination des peintres, des poètes et des musiciens. Rensselaer W. Lee l'utilise comme l'exemple de son désormais classique Ut Pictura Poesis, et rappelle les peintres importants, dont Poussin, qui ont traité ce sujet. Ce thème fut aussi celui du ballet de La Délivrance de 
Renaud, d'Étienne Durand (1617), des Amours déguisés de Benserade et Lully (1664). Quinault écrivit un Armide et Renaud (La Comédie sans comédie, 1655) et reprit le thème dans son livret d'opéra, thème pastoral, merveilleux et magique, propre au genre de l'opéra classique. Ellen Harris, dans son Handel and the Pastoral Tradition, a donc raison de souligner la nature spécifique de ce genre, aux diverses nuances :

Ceux qui étudient les traditions de l'opéra baroque doivent se rappeler que la polarité aristotélicienne entre la tragédie et la comédie ne tient pas longtemps. En fait le contraste se situe entre les différentes sortes de tragi-comédies - les plus importantes étant la pastorale et le drame héroïque, qui comprend des éléments à la fois sérieux et comiques $(1980: 2)^{4}$.

Armide n'a rien de comique, mais elle est un personnage de la "pastorale héroïque ». Dans la tragédie racinienne, comme dit Girdlestone, "le climat est étouffant ", alors que "l'air de fête que tous ces opéras respirent à des degrés divers » se déploie " en plein air ", " dans un beau temps perpétuel » (1972: 120-121). Le monologue d'Armide présente un mélange des styles qui devait frapper les contemporains : le contraste très dense entre le style sublime, héroïque, et le style " tempéré » de la pastorale. Titon du Tillet nous le fait sentir dans sa description du jeu de Marie Rochois :

Dans quel ravissement n'étoit-on pas (...) de la voir le poignard à la main, prête à percer le sein de Renaud, endormi sur un lit de verdure! La fureur l'animait à son aspect, l'amour venait s'emparer de son cœur; l'une et l'autre l'agitoient tour à tour, la pitié et la tendresse leur succédoient à la fin, et l'amour restoit le vainqueur (1727: 792).

Armide mêle ainsi le romanesque, le pastoral et le tragique. C'est d'abord le style sublime du genre tragique, la " grande manière " " magnifique " des peintres (ces mots sont de Poussin), le " grand goût " qui donnera la théorie académique de l'emphase avec Le Brun. C'est le style " héroïque » de Roger de Piles (1708), mais aussi, chez les littéraires, le gravis stylus des commentateurs de Virgile et de l'Énéide, comme le style plutôt " pompeux " des discours des orateurs de la chaire et du barreau. Armide levant son poignard est un cas de style sublime. Armide tombant amoureuse de Renaud soupire dans le style "modéré ", " tempéré ", " médiocre », celui des Géorgiques, souvent difficile à distinguer du style simple des Bucoliques, où les bergers se lamentent. 
Le style sublime est " orné ", " animé, plein de mouvement, enrichi de figures, de toutes sortes de métaphores», dit le rhétoricien Lamy (1699 : 264). Le style modéré doit " être doux ", fait "d'un certain moelleux mêlé de délicatesse et de simplicité ", ajoute Charles Batteux (1775:107). Bon dramaturge, Quinault mêle les deux, les fait s'entrechoquer. Il joue avec la règle classique de l'unité et de la variété. On imagine bien son personnage comme un tableau mêlant, pour reprendre Roger de Piles et son Cours de peinture par principes au chapitre " du choix des attitudes", les contrastes, la diversité, "l'opposition des mouvements ", règle impérative de la danse et de la sculpture. La dramaturgie de Quinault et de Lully vise, au nom des règles et de la nature, à créer ce " tout-ensemble ", cette "opposition des lignes qui forment les objets, par laquelle ils se font valoir l'un l'autre " (Piles $1708: 102$ et 104). Ici, c'est déjà le "style coupé » "haletant " qui plaira tant à Diderot.

\section{L'action : la déclamation du discours pathétique}

L'action, c'est le théâtre de l'orateur, la dernière et indispensable partie de la rhétorique. Par elle, l'orateur finit de convaincre, car, si sa cause est juste, son ton sera juste, si les sentiments qu'il veut soulever sont naturels, son expression sera claire. Dans l'action, celui qui parle, peint, joue ou chante, tire les passions de la nature confuse. Cicéron conseillait donc de trouver les justes signes pour exprimer ces vérités :

Sans doute la vérité toujours l'emporte sur l'imitation; toutefois si, à elle seule, elle suffisait dans l'action, l'art deviendrait absolument inutile. Mais comme les passions de l'âme, que l'action doit avant tout mettre en lumière ou imiter, sont souvent si confuses, qu'elles sont reléguées dans l'ombre et presque enfouies dans l'oubli, il faut les faire sortir de l'ombre et s'attacher aux traits saillants qui les mettent en relief (trad. Bornecque \& Courbaud 1930 : III, 89).

Le musicien, comme l'orateur, doit savoir lire ce dictionnaire, et choisir : "l'Orateur et le Musicien ont tous les deux le même dessein (...) Il veulent s'emparer des coeurs (...) Il leur est avantageux lorsque l'un a quelques notions des connaissances de l'autre ", disait Quantz (1752: 102). Le rôle de l'action est donc de mettre à jour ces passions par des signes qui les rendent fidèlement : sons, gestes, attitudes, couleurs, etc. La règle première, qui relie invention, élocution et action est donnée par l'Encyclopédie; à l'article "déclamation »: "C'est au style 
à suivre la marche du sentiment; c'est à la déclamation de suivre la marche du style."

Je propose ici un relevé des principaux moyens techniques et expressifs dont disposait le chanteur pour rendre le "chantdéclamation " du récitatif pathétique. Je donnerai à cette technique l'appellation générale de prononciation expressive, en souvenir de la pronuntiatio des orateurs.

\section{A. La bonne articulation}

Bérard (1755 : 47) distingue la prononciation de l'articulation. Celle-ci est la diction nette des sons de la langue. Comme " l'art de bien dire est le premier pas vers le théâtre " (Riccoboni 1750 : 96), l'apprenti devra s'exercer d'abord à une articulation claire qui respecte les qualités de base du style (propriété, vérité, brièveté, naturel, simplicité, contraste, élégance, facilité). On voulait une prononciation correcte, parisienne, "éduquée ", qui tienne compte des e atones, des hiatus, des élisions et des liaisons, sans oublier les respirations correctes. Le récitatif doit donc être parfaitement perçu et compris : trop de chant l'obscurcit.

\section{B. La prononciation expressive}

Bérard écrit :

Comme la prononciation dans le Chant peut être envisagée comme une espèce d'imitation des choses dont les paroles sont signes, elle doit varier selon le caractère de ces dernières (1755: 102).

On peut donc relever les procédés suivants :

1) Le timbre de la voix : Loulié (1696:43) relève que le "son peut être aigre ou doux ». Mais les orateurs recommandent d'éviter les tons "criards " (Le Gras 1671:272), et Quintilien déconseillait les voix "dures " et "aigres " (Institution Oratoire, XI, 3). Les français, bien sûr aiment les belles voix, avec les nuances que Bacilly distingue entre les " belles " et les "bonnes" voix $(1679: 38)$, les "grandes", les "petites", les «fortes ou foibles, brillantes ou touchantes" (ibid. : 41). Mais Armide ne demande-t-elle pas toutes ces qualités à la fois ?

2) Les " sons à caractère " : afin de rendre "l'imitation des choses ", Bérard décrit les " sons à caractère ", c'est-à-dire une prononciation "dure », " obscure ", "étouffée », " douce», "claire" selon que le sentiment est pathétique ou tendre. 
Ce sera aussi « le redoublement des lettres dans tous les endroits marqués au coin de la passion » (1755: 95), c'est-à-dire l'accentuation plus ou moins forte des consonnes importantes contenues dans les mots expressifs. La force de "l'haleine " (l'expiration), mettra en valeur les " sons majestueux ", " violents ", " étouffés ", " entrecoupés ", ou bien "légers ». Imaginons la déclamation d'Armide tour à tour haletante et tendre, reprise et soulignée par leurs équivalents gestiques et physionomiques ...

3) Les " tons de la voix »: " les tons sont à moitié fournis dans les mots, il ne faut qu'un peu d'art pour les en tirer " (Batteux $1775: 272$ ). Ces "tons de voix» sont donc les "inflexions affectives " (Larthomas $1980: 56)$ qu'on peut répertorier ainsi : Intervalles : Masson (1930) a bien rendu compte des intervalles expressifs dans le récitatif de Rameau. Le monologue d'Armide est dans la même veine : quinte montante ("enfin »), ou quarte ("frappons"). Cette direction expressive y est évidente, jusqu'à l'horizontalité du début du troisième vers («le charme du sommeil ").

Hauteur : comme chez ses prédécesseurs italiens, Lully respecte l'expressivité de la hauteur dans le gamut : l'aigu est propre aux sentiments agités ( "Vengeons-nous "), le registre moyen aux sentiments tranquilles (" je soupire»), le grave aux lamentations, ou à la " somnolence ", ou aux doutes ("Quel trouble me saisit? "). Le monologue d'Armide favorise l'agitation et va surtout dans l'aigu, registre préféré de la Champmeslé, dont Lully notait les inflexions : " la fameuse Champmeslé (...) avoit une voix sonore et fort éclatante dans le haut. Les tons élevés lui étoient favorables, elle les employait avec succès " (Riccoboni $1750: 18)$.

Accents : Morier (1975: "accent ") et Larthomas (1980:62) s'entendent pour distinguer les accents « normal », " intellectuel » et "émotionnel » du français. L'accent émotionnel déplace l'accent normal ("puissance ": la première syllabe est notée une tierce plus haut que la deuxième), et tombe ainsi sur les parties faibles de la mesure. Les exclamations dont ce monologue est plein font partie de ces jeux d'accentuation. Lully aime aussi déplacer les interjections sur des contre-temps ou des syncopes :

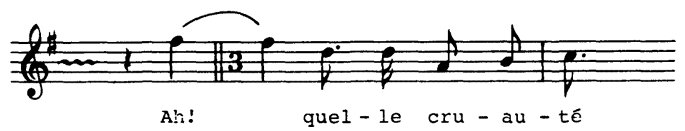


Ornements : plus que leur qualité décorative ou articulatoire (les tremblements et chutes de tierce des terminaisons féminines), la fonction expressive des ornements devrait être soulignée. Estève résume deux siècles en y voyant "l'âme de la déclamation» (1753:133). L'ornement n'est que la codification des inflexions expressives, et la lecture de leur nom seul chez Bacilly, Montéclair (1736) ou Bérard trahit déjà leur sens (du moins leurs rôles possibles, leur champ d'application). On sait que Lully mettait à la porte ceux qui en abusaient. Les ornements du monologue d'Armide sont économisés, mais efficaces. Le trille sur la deuxième syllabe de "puissance » consternait Rousseau (1753), ce à quoi Rameau répliqua par un argument d'ordre expressif : "Le trille fait beauté dans notre musique, surtout dans le cas présent, où il ajoute de la force au mot puissance, sur lequel porte tout le sens du vers» (1754:70).

Il serait peut être excessif d'utiliser ici les ornements à "effets spéciaux " de Montéclair (le "son glissé ", notre glissando), mais on aurait intérêt à essayer quelques-uns de ces " accents", "ports de voix ", ou "sanglots", les différentes " cadences » de Bérard, pour donner à la déclamation d'Armide une forte stylisation expressive.

4) Les figures : dans son Traité de la diction, Estève parle d'« images sensibles" $(1755: 23)$. Les traités de première et seconde rhétoriques rangent ces images sous le nom de figures. La question est très complexe, et je n'en donnerai qu'un bref aperçu.

Figures de mots : ce sont ces figures musicales plus ou moins stéréotypées qui cherchent à imiter le sens du mot ("ciel ", " enfer ", "gloire ", " chaînes "). Masson (1930) a donné une bonne idée de ces "tours de chant" chez Rameau.

Figures de passions:l'abbé d'Aubignac insiste sur leur importance dans le "poème dramatique ": «Il ne faut rien exprimer sur la scène qu'avec figures (...) Si la poésie est l'empire des figures, le théâtre en est le trône " $(1715: 312)$. Certains auteurs ont décrit leur expression. Ainsi, lorsqu'Armide s'adonne à la rage il lui faudra " une voix tonnante et quelquefois étouffée, mais toujours soutenue d'une extrème vigueur de poitrine " (Riccoboni $1750: 52$ ), une voix " âpre " de la colère "soutenue de l'espérance de se venger" (Grimares $1760: 92$ ). La tendresse aura bien sûr de la "douceur » et de la "finesse " (Riccoboni $1750: 46$ ), sa voix sera "flatteuse» (Grimarest $1760: 81)$. 
Les rhéteurs et les gens de théâtre aiment décrire ces figures de passions, nombreuses et difficiles à définir et à classer. Grimarest cite «l'interrogation, l'apostrophe, la prosopopée, l'antithèse, le serment, l'ironie, l'exclamation, l'epizeuxis et la gradation », car " toutes ont des tons qui leur sont particuliers » (1760 : 96). Lully devait se plaire à relever ces " gestes vocaux " (Larthomas $1980: 74$ ) : le monologue d'Armide est plein de ces exclamations, interrogations, apostrophes (premier vers), antithèses (" achevons ... je frémis »), réticences et ellipses ("qu'il éprouve toute ma rage ... Quel trouble me saisit»), gradations, etc.

C. Le rythme oratoire et les problèmes de tempo

La déclamation du vers français classique impose une mélodie particulière que Morier (1975) a étudiée en détail, et qui, par sa prosodie (arsis, thésis, repos, etc.), impose un débit particulier. La poétique de la bienséance éclaire d'une autre façon les problèmes de tempos et de changements de signes de mesure dont Lully parsème ses récitatifs. Quand certains s'adonnent à des spéculations d'ordre mathématique pour élucider les rapports métriques de ces signes (car les théoriciens se contredisaient) (Wold 1978; Fajon 1978), force leur est de conclure que "nous devons probablement traiter chaque cas en particulier » (Wolf $1978: 49$ ). Grimarest avait déjà la réponse :

L'on ne doit point battre la mesure, parce que l'acteur doit être le maître de son chant pour le rendre conforme à son expression et l'accompagnement doit aussi être assujetti à sa manière de chanter $(1760: 135)$.

Lécuyer dira plus tard que «la mesure [n'est] pas stricte dans la Scène ou dans le Monologue " (1769:22). Les traités de solfège et de théorie montrent que l'on plaçait d'abord la déclamation du texte sous la musique, indépendamment des valeurs. Par ailleurs, d'autres phénomènes entrent en jeu, comme les passages en récitatifs "mesurés", ou tout simplement les moments qui demandent plus de chant et moins de "récit» : " le charme du sommeil " est chanté, " le livre à ma vengeance " est déclamé. De même le cinquième vers ("par lui tous mes captifs ... ") relève du récitatif simple, "débité " : la note tenue qui le soutient en est une preuve.

Par ailleurs, d'autres particularités de la déclamation 
classique influencent le rythme et le tempo du récitatif : La quantité : Bacilly décrit longuement cette règle des quantités, des syllabes longues et brèves, et sera suivi par de nombreux auteurs au siècle suivant. Mais en dehors d'un certain nombre de mots où cette règle va encore de soi, la reconstitution de cette théorie artificielle de "musique mesurée à l'antique " pose aujourd'hui des problèmes : Bacilly n'est pas toujours clair, et ses successeurs ne sont pas toujours d'accord entre eux. La règle des quantités sonne maintenant faux si elle n'est pas reliée à l'expression, ou tout simplement à ce que Chailley (1974) décrit comme une tendance naturelle du langage à trouver des appuis plus ou moins réguliers à l'intérieur des mots. Ainsi dans " ennemi ", on pourra allonger la première syllabe, puisque deux $n$ suivent le e (Bacilly), mais aussi parce que Bérard aurait conseillé de doubler le son $n$ pour des raisons expressives.

Les notes inégales: cette règle, interdite dans le récitatif simplement débité, peut réapparaître dans les moments plus " chantés " du récitatif-monologue : " le charme » (du sommeil), est noté pointé, et on peut faire une seconde inégalité sur " du " et "sommeil ", inégalité différente de la première, puisqu'on allongera doucement le $s$ (Bérard). Les moments expressifs commandent presque cette règle : "je frémis " (notes inégales, débit lent), "vengeons-nous" (notes égales, débit rapide).

Longueur des vers : le nombre de pieds dans un vers pouvait affecter leur débit : "le grand vers a plus de majesté; le petit a ordinairement plus de feu ou de douceur " (Batteux $1775: 232$ ). Cette règle coïncide avec la théorie de la rythmopoeie qui, disait Mersenne, "consiste à connoistre et à choisir les pieds ou les mètres, et les vers qui sont propres pour exprimer les passions " (Traité des consonnances, in 1636-37:401).

Dans le récitatif d'Armide, on découvre ainsi que les vers les plus courts sont placés aux moments importants du discours : le premier ("enfin ... ") marque l'impatience d'Armide (elle attend ce moment depuis deux actes), le quatrième raccourcit le débit ("le livre à ma vengeance ») après le lent "charme du sommeil ", le sixième ("qu'il éprouve ...") est un brusque emportement, le neuvième ("frappons") est au moment de la plus extrême tension, c'est pourquoi le suivant prendra un très long temps, avec ses deux antithèses.

Longueur des mots : le nombre de syllabes d'un mot peut avoir une importance expressive : une suite de monosyllabes peut, en fonction des paramètres énoncés plus haut, jouer un rôle 
différent de celui d'un mot très long. Les dix premiers vers du monologue d'Armide n'ont qu'un mot expressif de quatre syllabes : "invincible », qu'on sera porté à prononcer avec plus de lenteur et d'emphase que "esclavage " du vers suivant (les valeurs sont d'ailleurs plus longues).

\section{L'accompagnement}

Rameau a suffisamment commenté ce récitatif de Lully et l'expressivité de l'harmonie pour qu'on y revienne. On pourrait disserter sur le bien-fondé de ses théories, mais arrivé le moment d'interpréter Armide, il n'est plus temps de mettre en scène des hypothèses. L'expérience pratique montre vite que le seul fait de suivre Rameau à la lettre, est efficace et donne une forte impression de cohérence. Les tensions, les détentes, les mouvements expressifs d'un accord à l'autre, le " jeu chromatique " de Lully, dit Rameau, tout contribue à une extrême densité de cette action déclamatoire exactement contenue dans l'harmonie et soutenue par le continuo. La pantomime vocale et gestuelle de la comédienne se reflète dans la pantomime de ses accompagnateurs, qui, en retour, la nourrit : articulations et arpèges du clavecin, gestique visuelle et sonore de l'archet, tel est le ballet d'une autre déclamation qui enveloppe et amplifie celle du personnage principal.

\section{Vers une disposition affective : l'ordre dans le désordre des passions}

Le danger habituel de cette esthétique est de n'être qu'une accumulation de détails. Barthes décrivait ainsi, et avec raison, cet « art bourgeois " qui

(...) croit que la vérité d'un ensemble ne peut être que la somme des vérités particulières (...) Ensuite de quoi, on attribue une signification emphatique à la plus grande quantité de détails (1963: 128).

Là probablement réside ce qui nous sépare des comédiens et des chanteurs du temps de Lully : l'emphase mise sur le plus petit niveau (le mot, la syllabe), rehaussée de cette déclamation chantante.

Nos goûts vont vers une autre simplicité; nous sommes portés à considérer la "forme ", la " cohérence ", la " structure ". Pourtant les classiques souhaitaient cette cohérence, cette hiérarchie des moyens et des effets. L'abbé d'Aubignac parle de 
la nécessité de "régler un discours pathétique» (1715:298). Il y faut des choix, une disposition particulière. Le monologue d'Armide est la peinture d'une confusion affective : ce désordre affectif réclame un ordre, sous peine de dérouter l'auditeur par un réalisme incohérent. Cet ordre naîtra de la concentration des effets dramaturgiques dont parle Larthomas, et de la disposition rhétorique du discours.

\section{A. Mettre en valeur l'essentiel}

Larthomas souligne ces "appuis du discours » qui font " appel à l'attention sans cesse défaillante de l'interlocuteur" (1980 : 282) : Armide est pleine d'exclamations et de silences qui tiennent le spectateur en haleine. Le même auteur décrit aussi ces "éléments de présentation [qui] précèdent généralement l'élément à mettre en valeur " (ibid. : 283) : c'est le " enfin » du premier vers, qui, après une longue attente, nous indique qu'il "va se passer quelque chose ". C'est donc l'art de " mettre en valeur l'essentiel », conclue Larthomas (ibid. : 284), une dramaturgie de la parole bien connue des gens de théâtre. Remond de Sainte-Albine parlait de la nécessité de " préparer et de graduer les grands mouvements " (1749: 197). Lacépède conseillait au musicien de "choisir (...) le mot le plus expressif, celui qui est comme un petit abrégé de la phrase (...) et sur lequel l'acteur pourra, par un seul geste ou par un seul ton de voix, peindre un petit tableau du sentiment qui l'anime " (1785, II : 43-44).

Il faut donc choisir, organiser. D'Aubignac recommande de trier parmi les figures de passions, afin de ne conserver " que les plus belles». Il préconise l'économie des énergies qu'elles renferment : "il faut bien prendre garde à ne pas consumer toutes les forces d'une passion, dès que l'on commence à la toucher" (1715:307). Si on veut suivre les " mouvements de la nature ", ce sera avec ordre, avec des liaisons, des transitions, des contrastes. Avec d'Aubignac, comme avec bien d'autres, les figures de passions deviennent finalement des figures de construction, les unes et les autres n'étant pas toujours faciles à distinguer dès qu'on veut rendre un texte expressif. Ces figures de construction permettront ainsi de " réformer ce que la nature a de défectueux en ses mouvements; et par la variété sensible des figures on garde une ressemblance du désordre de la nature " (Aubignac $1715: 310-311$ ). 
B. La stratégie oratoire

Il s'agit donc d'un "tout-ensemble » qui est une forme construite à partir d'éléments dynamiques, dramaturgiques et oratoires. La place des procédés destinés à soulever l'émotion de l'auditeur est capitale. La disposition rhétorique (deuxième partie de l'art du discours) est une stratégie qu'il faut soigneusement calculer : " la force et la beauté du discours (...) consistent principalement dans un arrangement juste et naturel de toutes les parties qui le composent" (Bretteville $1701: 102$ ).

Cette disposition se retrouvera dans le discours, ou monologue pathétique. Il lui faut un exorde pour capter l'attention des auditeurs (" enfin il est en ma puissance"). Viendra ensuite le discours lui-même, l'ipsum corpus carminis, lieu d'exposition des arguments et de délibération, exposé des "réfutations" et des "confirmations", intellectuelles ou affectives. Armide prononce un discours pathétique qui tient en partie à une sorte de délibération ordonnée, où les figures suivent avec logique l'évolution psychologique. Les six premiers vers exposent le sujet, les quatre suivants amènent des sentiments (des arguments) contraires. La suite ne sera plus qu'un commentaire sur le bouleversement qui vient d'arriver.

Enfin, il faut une péroraison. "Les discours pathétiques ne doivent pas finir comme ils commencent», dit d'Aubignac. Celui de Quinault se termine par un air (« venez, venez seconder mes désirs, démons ... "), qui est, cette fois, vraiment chanté. Car le chant amplifie ce moment affectif par excellence du discours oratoire : " la péroraison (...) doit renfermer tout le grand et tout le sublime de l'éloquence (...) La péroraison n'est que pour le cour" (Bretteville $1701:$ 179).

\section{La rupture avec la norme}

Rompre avec la norme, c'est déjà faire une figure, dit-on. Si on veut bien considérer la norme comme l'énonciation simple et neutre, ce qui est bien approximatif, on pourra ainsi mieux comprendre comment choisir parmi tous ces procédés de détails. Leur accumulation serait déjà une forme de norme, leur absence une autre. La concentration des effets repose sur cette stratégie des ruptures avec le prévisible. Il se peut que les mauvais acteurs aient négligé cette règle au profit d'une emphase égocentrique. Il se peut que des comédiens trop dociles aient manifesté un respect ennuyeux des règles. Mais quand les auteurs se mêlent d'avoir du génie, ils font fi des règles, et s'en jouent 
publiquement. Les six premiers vers du monologue d'Armide ont tout de la "période carrée ", "composée " de quatre membres. C'est presque la belle période classique qui porte à la déclamation mélodique et académique :

En prononçant une période entière on élève la voix jusqu'à la moitié de la sentence, et elle se rabaisse dans l'autre moitié : ces deux parties qui sont appelées arsis et apodosis doivent se répondre par leur égalité (Lamy 1699 : 200).

Par chance, Quinault et Lully veillent. La période reste inachevée et Armide tombe amoureuse.

\section{Pour conclure : Lully est-il jouable?}

L'esthétique de la poesia musicale engendre donc une technique expressive, une codification serrée des moyens, un répertoire de gestes sonores et visuels qui sera encore utilisé jusqu'au début du $\mathrm{XX}^{\mathrm{e}}$ siècle. Stanislavski, au début de ce siècle, rejettera ce qui était devenu un magasin d'accessoires et sentait son cabotinage, le "jeu mécanique ", " outré ", " comme, par exemple, le fait de porter la main sur le cour pour exprimer l'amour, ou d'ouvrir la bouche pour faire voir qu'on va mourir » (1979: 31).

C'est que, par la force des règles de la bienséance, la technique tenait finalement lieu d'expression, et l'acteur, comme l'auditeur, n'avait qu'à suivre un code gestique et déclamatoire considéré comme établi. Quand Gluck écrit un récitatif accompagné sur le même texte que l'Armide de Quinault, il ne fait que suivre la même esthétique : la musique est différente, mais l'essentiel demeure, et les commentateurs utilisent les mêmes termes. L'expression peut changer dans les détails, elle reste " universelle ": Remond de Saint-Mard, à propos de Lully, défend ce point de vue :

Un sentiment s'exprime par-tout à peu près de même (...) En tous tems et en tous pays, un sentiment tendre s'exprimera d'une manière tendre. Par-tout un mouvement de colère sera rendu d'une manière vive. (1741: 86).

"Qu'on mette Armide en Chine ", continue-t-il, et sa manière de dire sera la même. La déclamation reposait, disait-on (naïvement ?) sur des règles immuables. Lully, dit Grimarest, est le maître de cette "exactitude " de la déclamation. L'acteur et l'auditeur se reposeront avec tranquillité sur elle : 
Il a conservé une proportion convenable avec les règles de la Déclamation. Ainsi l'acteur qui exécute n'a point de peine, il se plaît à chanter ces endroits; et celui qui écoute ne perd point le sentiment, et jouit en même tems du plaisir d'entendre d'excellente musique : il la saisit dès la première fois qu'il l'entend, parce qu'elle est dans la nature (1760:126).

Barthes a parfaitement défini cette technique à propos de l'alexandrin racinien :

Comme dans tout théâtre codifié, la règle se substitue ouvertement à la subjectivité, la technique à l'expression. Il y a bien des rapports entre la rigueur de la règle classique et la syntaxe impérative des symboles gestuels et vestimentaires dans le théâtre oriental : l'une et l'autre sont là pour épuiser l'acteur, pour substituer son savoir à son inspiration. Imagine-t-on un acteur chinois qui combinerait indiscrètement le respect d'une symbolique ancestrale et une expressivité personnelle empruntée à notre naturalisme (1963: 138-139) ?

La reconstitution de la déclamation classique pourrait bien paraître insupportable, emphatique, affectée, à nos oreilles habituées à un autre "naturel ", s'il n'y avait le chant et cette "symbolique » codifiée.

Remond de Saint-Mard lui-même reconnaissait que l'actrice Journet ne valait pas la chanteuse Le Couvreur (1741:9). Le chant sauve cette déclamation passée de mode qu'on peut encore entendre sur les rouleaux enregistrés par Sarah Bernard, à condition que la technique moderne de la "grande ligne " et du masque ne vienne pas créer une ambiguïté : la poesia musicale ne supporte pas le bel canto. Lorsque Barthes écrit qu'« à la limite l'alexandrin dispense l'acteur d'avoir du talent " (1963 : 138), il nous suggère que l'interprétation du récitatif d'Armide est une entreprise hautement stylistique. Le chanteur est d'abord un comédien qui combine les éléments sonores, dynamiques, " actifs » d'une dramaturgie codifiée. À lui ensuite d'y mettre "du feu », des "entrailles », du " génie ». Un monologue pathétique comme celui d'Armide suggère une action qui pourra nous émouvoir si elle nous étonne par une recherche spécifiquement dramaturgique. Elle aura alors tous les atouts de la nouveauté. 


\title{
ANNEXE
}

Jean-Baptiste Lully : Armide (1686), d'après d'Alembert, Éléments de Musique (1752)

\author{
ARMIDE, TRAGE DIE.
}
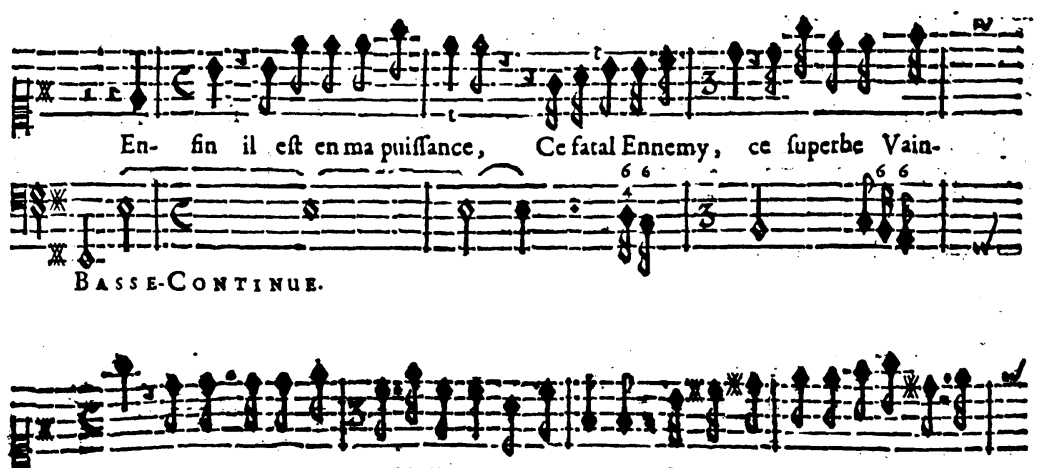

queur. Le tharmedu form-meil le livre.̀ ma vengeance; Je vais percer fon invincible

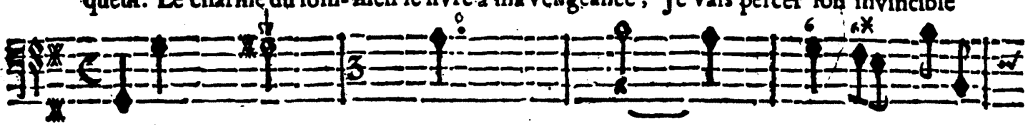

B Asse. Continue.

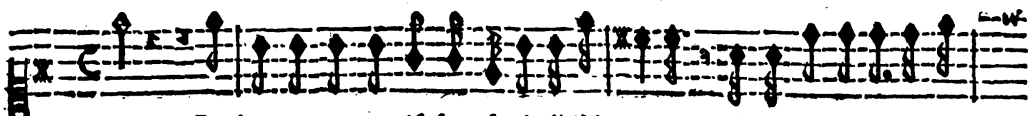

cocur. Par luy tous mes captifs font forris d'Aclavages $x$ Qu'il ciprouve route me

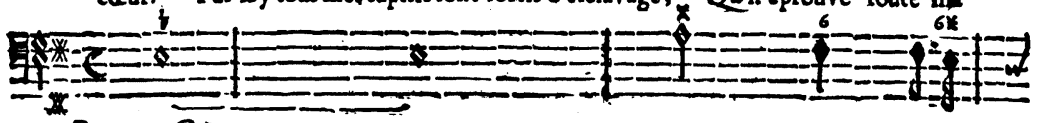

BASSE-CONTINUEV
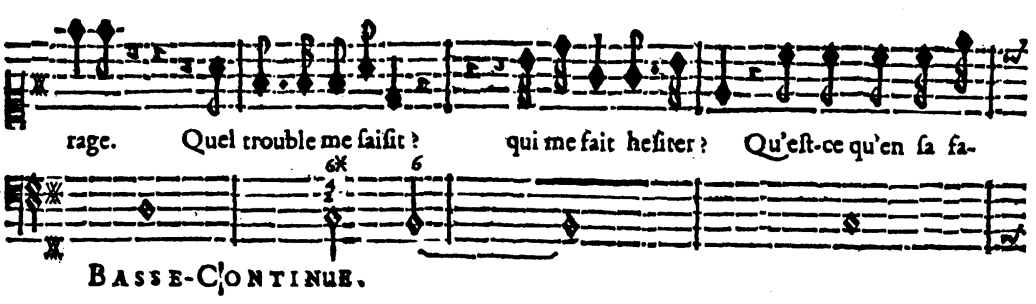

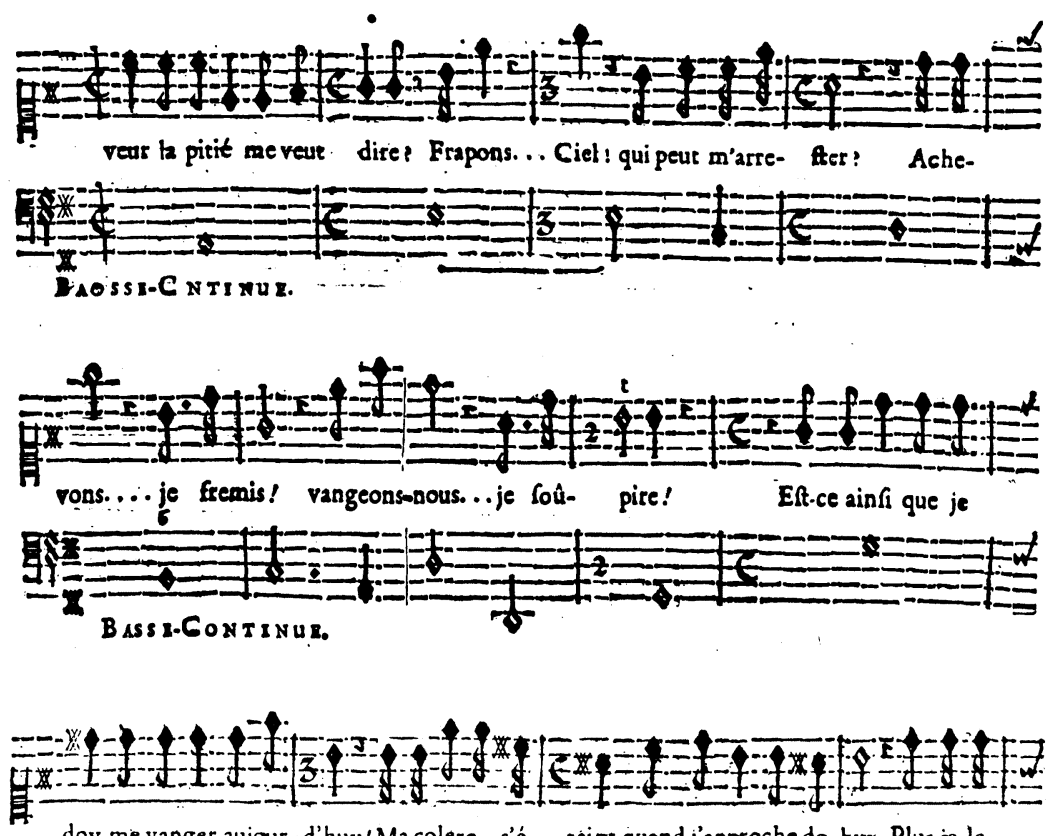

doy me vanger aujour-d'huy! Ma colere s'é- teirx quand j'approche de. huy. Plus je le
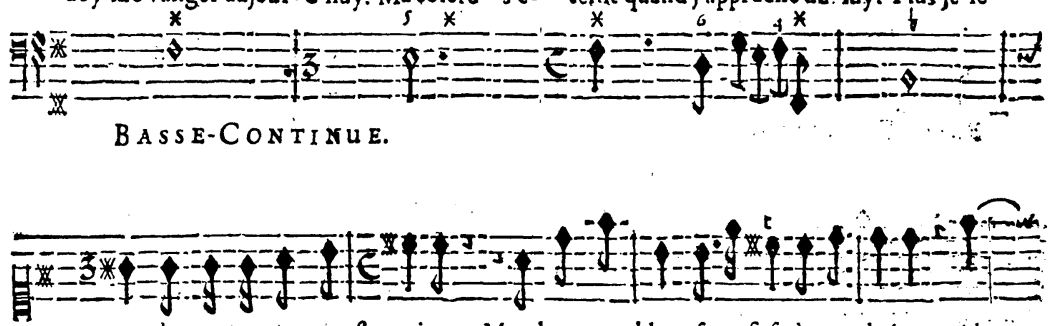

voy; plus ma vengeance eft . vaine; Mon brastremblane fe refufe à ma haine. Ah!"
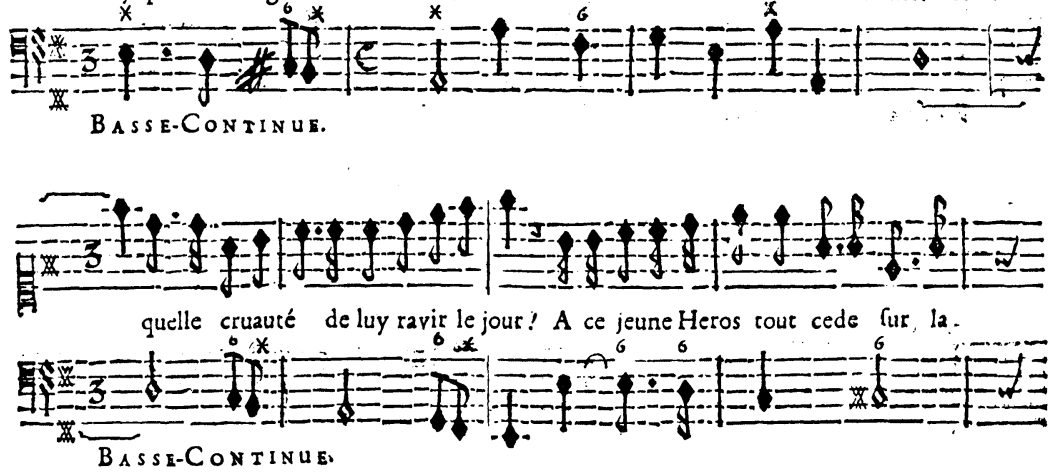


\section{ACTE SECOND:}
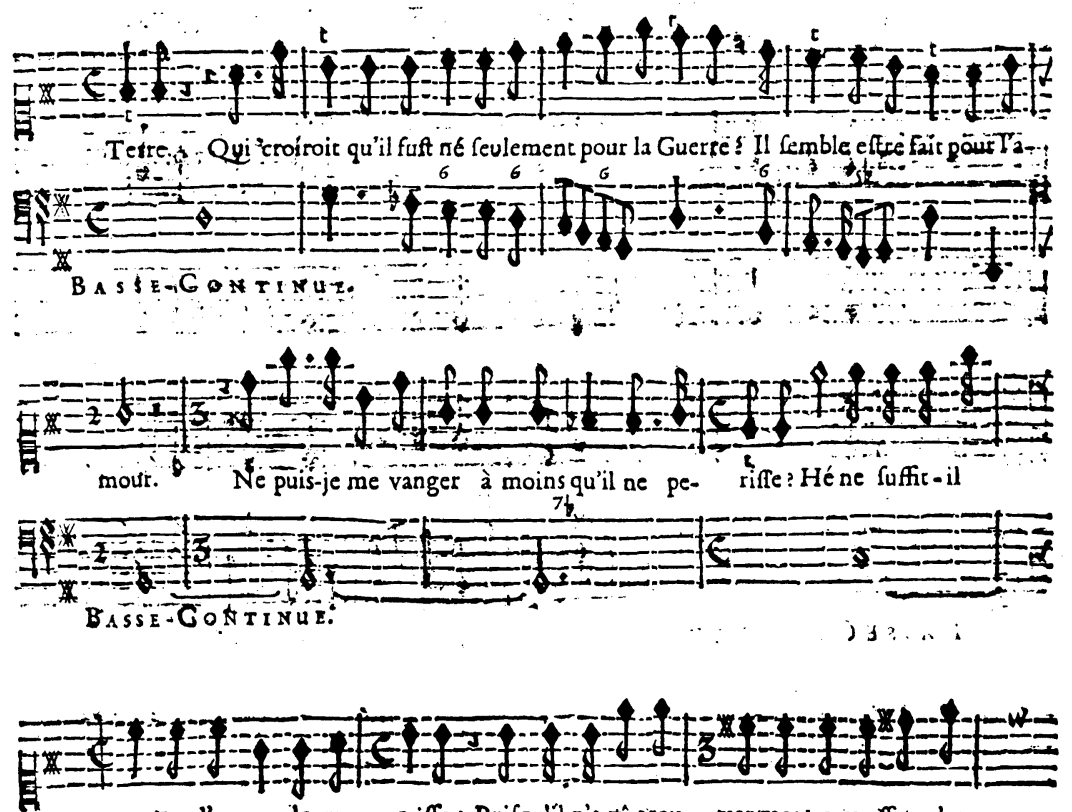

pas quet'amour le pa- niffe? Puifqu'il n'a pû trou- ver mes yeux affez char-
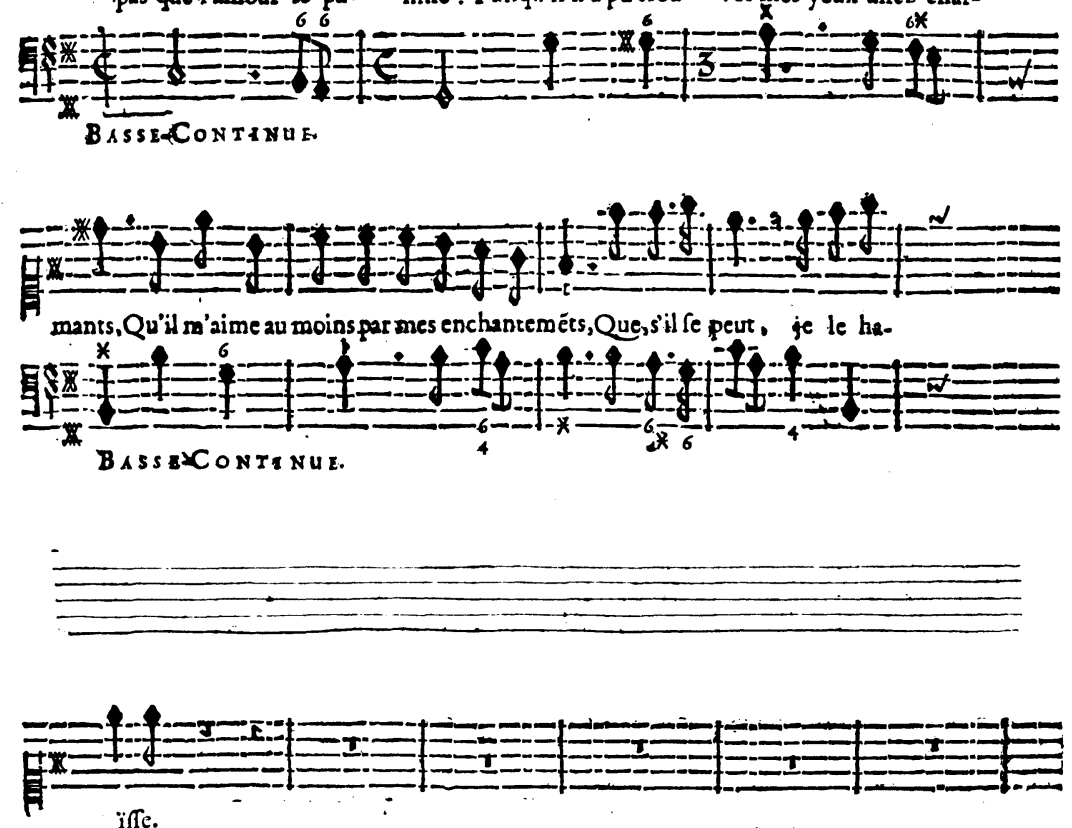

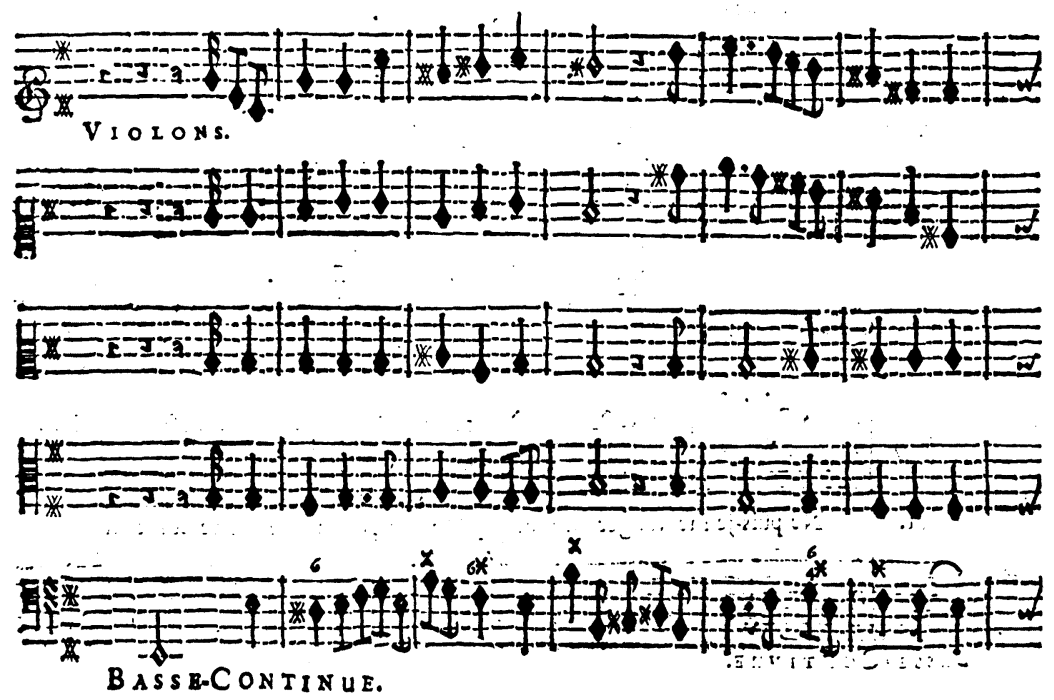


\section{NOTES}

1. "La déclamation de Lulli est une mélopée si parfaite que je déclame tout son récitatif en suivant ses notes, et en adoucissant seulement les intonations; je fais alors un très grand effet sur les auditeurs, et il n'y a personne qui ne soit ému " (Voltaire, lettre à Chabanon, 18 décembre 1767; cité par Peyronnet [1974:76]).

2. Si on passe sous silence les tentatives du ballet de cour; cf. La Laurencie (1930) et McGowan (1978).

3. On peut se reporter à l'ouvrage le plus connu du XVIII siècle traitant de l'allégorie : Cesare Ripa, Iconologia (1603), réédité avec commentaires et gravures par J.G. Hertel, 1758-1760.

4. "The student of baroque operatic traditions must remember that the Aristotelian polarity of tragedy and comedy no longer obtains. Instead the contrast is between various kinds of tragi-comedy the most important being pastoral and heroic drama - which include both serious and comic elements".

\section{RÉFÉRENCES}

AUBIGNAC, F.H., Abbé d'

1715: La Pratique du théâtre. 2 vol. Amsterdam : J.-F. Bernard. BACILLY, B. de

1679: Remarques curieuses sur l'art de bien chanter. Paris: Ballard.

BARRAULT, J.-L.

1946: Mise en scène de Phèdre. Paris : Seuil.

BARNETT, D.

1977/78 : "The Performance Practice of Acting : The Eighteenth Century ", Theatre Research International, II/3, 157-186; III/1, 1-19; III/2, 79-93.

BARTHES, R.

1963 : Sur Racine. Paris : Seuil.

BATTEUX, Abbé C.

1775: Les Beaux-Arts réduits à un même principe (1746) suivi du Cours de belles-lettres (1747-1748) et De la construction

BÉRARD, J.-B. oratoire (1763). Paris. $5^{\mathrm{e}}$ ed.

1755: L'Art du chant. Paris : Dessaint et Saillant. BRAY, R.

1966 : Formation de la doctrine classique. Paris : Nizet. 
BRETTEVILLE, Abbé É.D. de

1701: L'Éloquence de la chaire et du barreau. $2^{\mathrm{e}}$ éd. Lyon : Guillimin.

CHAILLEY, J.

1974 : "Apport du vocal et du verbal dans l'interprétation de la musique française classique ", in L'interprétation de la musique française aux XVII et XVIII' siècles. Paris : CHIFLET, L. Éditions du Centre National de la Recherche Scientifique.

1659: Essai d'une parfaite grammaire de la langue françoise.

\section{CICERO} Paris : P. Maugé.

1930 : De Oratore. Trad. H. Bornecque et B. Courbaud. Paris : Les Belles-Lettres.

DORAT, C.-J.

1766: La Déclamation théâtrale. Paris : S. Jorry. DUVIGNAUD, J. ESTĖVE, P.

1970 : Spectacle et Société. Paris : Denoël.

1753 : L'Esprit des beaux-arts. Paris : C.-J.-B. Bauche.

1755 : Traité de la diction. Paris : Duchesne.

FAJON, R.

1978 : "Propositions pour une analyse rationalisée du récitatif FORMEY, J.-H.-S.

de l'opéra lullyste ", Revue de musicologie, LXIV/1, 55-75.

1763 : Principes élémentaires des belles-lettres. Paris. GIBERT, B.

1730/49: La Rhétorique, ou les Règles de l'éloquence. Paris: C.-L. Thiboust.

GIRDLESTONE, C.

1972: La tragédie en musique (1673-1750). Genève-Paris : Droz.

GRIMAREST, J.-L. Le Gallois, Sieur de

1707 : Traité du récitatif. Paris : J. Le Fève.

GROS, E.

1926 : Philippe Quinault, sa vie et son ouvre. Paris : Librairie Ancienne Honoré Champion.

HARRIS, E.

1980: Handel and the Pastoral Tradition. Londres: Oxford University Press.

LACÉPÈDE, B.-G.-É de La Ville, Comte de

1785 : La Poétique de la musique. 2 vol. Paris : Impr. de Monsieur.

LA LAURENCIE, L. de

1930 : Les Créateurs de l'opéra français. Paris : Arcan.

LAMY, B.

1699 : La Rhétorique, ou l'Art de parler. Amsterdam. 
LARTHOMAS, P.

1980: Le langage dramatique. Paris: Presses Universitaires de France.

LAZARE, M.

1980 : Le théâtre en France au XVI siècle. Paris : Presses Universitaires de France.

LE CERF DE LA VIÉVILLE de FRENEUSE, J.-L.

1705/06 : Comparaison de la musique italienne et de la musique

LÉCUYER.

française. $2^{\mathrm{c}}$ éd. Bruxelles : F. Foppens.

1769: Principes de l'art du chant. Paris : L'Auteur.

LE GRAS.

1671: La Rétorique [sic] française. Paris.

LOULIÉ, É.

1696 : Éléments ou principes de musique. Paris : Ballard.

McGOWAN, M.

1978: L'art du ballet de cour en France, 1581-1643. Paris : Éditions du Centre National de la Recherche Scientifique.

MASSON, P.-M.

1930: L'Opéra de Rameau. Paris : H. Laurens.

MERSENNE, M.

1636/37 : Harmonie universelle. Paris : S. Cramoisy.

MONTÉCLAIR, M de [pseud. de M. Pinolet]

1736 : Principes de musique. Paris : Boivin.

MORELLET, Abbé A.

1759: De l'expression en musique. Paris.

MORIER, $\mathrm{H}$.

1975 : Dictionnaire de poétique et de rhétorique. Paris : Presses Universitaires de France.

NATTIEZ, J.-J.

1983 : Tétralogies - Wagner, Boulez, Chéreau. Paris : Christian Bourgois.

NEWMAN, J.

1979: Jean-Baptiste de Lully and his Tragédies Lyriques. Ann Arbor, Mich. : UMI Research Press.

PEYRONNET, P.

1974 : La mise en scène au XVIII" siècle. Paris : Nizet.

PILES, R. de

1708: Cours de peinture par principes. Paris : J. Estienne.

PURE, Abbé M. de [pseud. Gélasire]

1668 : Idées des spectacles anciens et nouveaux. Paris : M. Brunet. QUANTZ, J.-J.

1752: Essai d'une méthode pour apprendre à jouer de la flûte traversière. Berlin : C.F. Voss. 
RAMEAU, J.-P.

1754: Observations sur notre instinct pour la musique. Paris : Proult fils.

RÉMOND de SAINTE-ALBINE, P.

1749 : Le Comédien. Paris : Vincent fils.

RÉMOND de SAINT-MARD, T. de

1741: Reflexions sur l'opéra. La Haye : J. Néaulme.

RICCOBONI, F.

1750: L'Art du théâtre. Paris : C.-F. Simon et Giffart fils.

ROUSSEAU, J.-J.

1753 : Lettre sur la musique française. Paris ; rep. in Écrits sur la musique. Paris : Stock-Musique (1979), 251-323.

SCHERER, J.

1950: La dramaturgie classique en France. Paris : Nizet.

SNYDERS, G.

1968: Le goût musical en France aux XVII' et XVIII' siècles. Paris : J. Vrin.

STANISLAVSKI, C.

1979 : La formation de l'acteur. Paris : Payot.

TITON DU TILLET, É.

1727, 1732, etc. : Le Parnasse françois. Paris : J.-B. Coignard fils. WOLF, R.P.

1978: "Metrical Relationship in French Recitative of the Seventeenth and Eighteenth Centuries ", Recherches sur la musique française classique, XVIII, 29-49. 